\title{
INVESTIGACIONES
}

\section{¿Por qué Hago Evaluación Formativa en Educación Física? Relato Autobiográfico de un Docente*}

\author{
Why do I use Formative Evaluation in Physical Education? \\ A Eeacher's Autobiographical Account
}

\author{
Txema Córdoba Jiménez $z^{a}$ Víctor Manuel López Pastor ${ }^{b}$, Enric Sebastiani Obrador \\ ${ }^{a}$ FPCEE Blanquerna, Universitat Ramon Llull, Telf.: (34) 932533000 \\ Correo electrónico: josemariacj@ blanquerna.url.edu

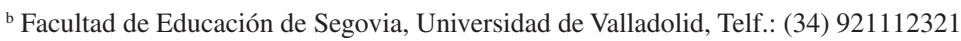 \\ Correo electrónico: vlopez@mpc.uva.es \\ ${ }^{c}$ FPCEE Blanquerna, Universitat Ramon Llull, Telf.: (34) 932533005 \\ Correo electrónico: enricmariaso@blanquerna.edu
}

\begin{abstract}
RESUMEN
Este estudio describe el proceso de evolución de un docente de educación física desde la perspectiva de los sistemas de evaluación que ha vivido como alumno y que ha utilizado como maestro. Mediante el relato autobiográfico, se analiza la evolución personal y profesional del autor, los motivos para empezar a utilizar un sistema de evaluación formativa y el proceso en la implantación de dicho modelo. A lo largo del relato se puede observar cómo pasa de un modelo de evaluación sumativa y calificativa, fruto de su experiencia como estudiante y su formación inicial, hacia un modelo que toma como base la evaluación formativa y la práctica reflexiva. Este modelo de evaluación, al servicio del aprendizaje del alumno y de sus consecuencias en la sociedad, se presenta como un nuevo reto coherente con la educación del siglo XXI.
\end{abstract}

Palabras claves: evaluación formativa, educación física, desarrollo profesional, relato autobiográfico, práctica reflexiva.

\section{ABSTRACT}

This study describes the evolution of a physical education teacher from the perspective of the assessment systems that he experienced as a student and that he has used as a teacher. Through an autobiographical account we analyze the personal and professional evolution of the author, the reasons for starting to use a formative assessment system, and the process of implementing this model. Throughout the narration, a transition can be observed from a model of summative and qualifying assessment, the result of the author's experience as a student and his initial training, to a model based on formative assessment and reflective practice. This model of assessment, which is practiced in the service of the student's learning and its consequences in society, is presented as a new challenge that is consistent with education in the 21 st century.

Keywords: formative assessment, physical education, professional development, autobiographical narration, reflexive practice.

Este estudio se ha llevado a cabo dentro del proyecto de I+D+i: "Las competencias docentes en la formación inicial del profesorado de educación física”. Convocatoria de noviembre de 2013 del Programa Estatal de Investigación, Desarrollo e Innovación Orientada a los Retos de la Sociedad, en el marco del Plan Estatal de Investigación Científica y Técnica y de Innovación 2013-2016. Referencia: EDU 2013-42024-R. Duración: 3 años (2014-2017). 


\section{INTRODUCCIÓN}

En el proceso educativo se ha venido utilizando tradicionalmente un sistema de evaluación sumativa donde se califica y clasifica al alumnado para cumplir con las exigencias burocráticas de la administración educativa. Desde hace algunas décadas se están extendiendo cada vez más propuestas que entienden la evaluación como una actividad para el aprendizaje, mediante la que se adquieren conocimientos y donde los alumnos tienen un papel protagonista (Álvarez-Méndez, 2001; Blázquez \& Sebastiani, 2016; Bonsón \& Benito, 2005; Brown \& Glasner, 2003; López-Pastor, 2006, 2009; López-Pastor \& PérezPueyo, 2017; López-Pastor et al., 2013; Pérez-Pueyo, 2005; Sanmartí, 2007; Santos-Guerra, 2003). Las principales características de estas propuestas de evaluación son (1) que están al servicio de los que se forman y de la práctica educativa; (2) que son democráticas, ya que requieren de la participación de todos los sujetos que se ven afectados del hecho educativo; (3) que forman, motivan y orientan el proceso de aprendizaje, y (4) que son transparentes y garantizan el conocimiento de los criterios utilizados.

En Educación Física (EF), el modelo tradicional de evaluación se ha venido basando en la utilización de test de condición física y de habilidades motrices con el fin de calificar al alumnado al final de cada trimestre (Hernández \& Velázquez, 2004; Herranz, 2013; López-Pastor, 1999, 2006). En las dos últimas décadas el inconformismo con ese modelo ha llevado a muchos profesionales a poner en práctica propuestas de evaluación orientadas al aprendizaje que permiten al alumno asumir responsabilidades y tomar decisiones en estos procesos (Blázquez, 2017; Córdoba, 2015; Hernández \& Velázquez, 2004; Herranz, 2013; Jiménez \& Navarro, 2008; López-Pastor, 1999, 2006; López-Pastor \& Pérez-Pueyo, 2017; López-Pastor et al., 2008; Moreno-Murcia, Vera \& Cervello, 2009; Pérez-Pueyo, 2005; Santos \& Fernández, 2009; Sebastiani, 1993; Sicilia et al., 2006; Velázquez-Callado, 2006). Muchos de estos autores exponen prácticas de evaluación formativa diseñadas para el beneficio de alumnos y profesores, tanto en el aprendizaje como en la práctica, sin carácter sancionador, y que se desarrollan de manera sistemática a lo largo de todo el proceso educativo. El alumnado acostumbra a participar en el proceso de evaluación, ya sea evaluándose a sí mismo (autoevaluación), evaluando a compañeros (coevaluación o evaluación entre iguales) o manteniendo procesos dialógicos con el profesorado respecto al proceso de aprendizaje que tiene lugar (evaluación compartida).

En este contexto, los objetivos de este estudio son (a) describir el crecimiento profesional y personal de un maestro de primaria en la temática de la evaluación en $\mathrm{EF}$, mediante un proceso autoreflexivo; (b) reflejar cómo la manera que tiene el docente de entender la evaluación contribuye de forma simultánea y sinérgica al desarrollo de su identidad personal y profesional, y (c) analizar los factores que influyen para que un maestro aplique en su práctica diaria sistemas de evaluación formativa.

\section{METODOLOGÍA}

Este trabajo está enmarcado dentro del paradigma de investigación educativa sociocrítica (Latorre, Del Rincón \& Arnal, 1996), que entiende que toda investigación educativa debe estar unida a un compromiso político orientado a la búsqueda de relaciones más justas e igualitarias entre las personas y entre los grupos sociales (Barba, González \& BarbaMartín, 2014; López-Pastor, 1999). 
Se utiliza el relato autobiográfico como metodología cualitativa para desarrollar procesos de reflexión y toma de conciencia del paradigma docente propio (González \& Barba, 2013; Sparkes \& Devís, 2007). Para ello, el instrumento es la historia de vida, mediante la cual el protagonista relata los acontecimientos más significativos de su propia vida alrededor de una temática, describiendo hechos y situaciones con las que construye su identidad profesional y otorga sentido a su vida en un momento dado (Barba, 2011; Bolívar, Domingo \& Fernández, 2001; González \& Barba, 2013; Pujadas, 1992). Este instrumento permite analizar las transformaciones sociales y singulares de los protagonistas y establecer relaciones con los contextos de vida profesional y social (Josso, 2014). Para Ruíz (2012), los objetivos que justifican la utilización de la historia de vida son los siguientes: (a) captar la totalidad de una experiencia biográfica en tiempo y en espacio; (b) captar la ambigüedad y el cambio, las dudas, las contradicciones y las vueltas atrás que se experimentan a lo largo de los años; (c) captar la visión subjetiva con la que uno se ve a sí mismo y al mundo; (d) descubrir las claves de interpretación de fenómenos sociales a través de la experiencia personal.

El papel del investigador es desarrollar el relato desde un punto de vista propio, sin pretensión de mostrar verdades objetivas, sino interpretaciones subjetivas que reflejan cómo piensa, siente y actúa. Su característica inherente es la expresión de sus propias actitudes desde una actitud crítica contra el poder hegemónico establecido (Barnett \& Parry, 2014; Chomsky, 2007). Este proceso de autoformación y transformación existencial que supone la historia de vida pretende encontrar vías de renovación social, educativa y política para el mañana (López-Górriz, 2007). Constituye un instrumento idóneo para la construcción del docente reflexivo, ya que promueve el acceso y la compresión del pensamiento en busca de una mejora de la práctica educativa.

En este relato autobiográfico se utiliza la evaluación como hilo conductor. Se narra, a partir de cuatro etapas ("El Alumno", "El Instructor", "El Maestro" y "El Educador"), cómo un maestro de EF va cambiando su perspectiva pedagógica, desde donde analiza y comprende su contexto educativo e interviene sobre él. Esta transformación discurre por los tres intereses constitutivos del saber que describe Habermas (1982), y que Grundy (1998) fundamenta en la teoría del conocimiento del currículum: (1) el técnico, preocupado en la reproducción epistemológica y metodológica de determinadas disciplinas; (2) el práctico, basado en la experimentación y la comprensión consensuada de significados; y (3) el emancipador, centrado en potenciar la autonomía, la responsabilidad y la autorreflexión.

Las dos primeras etapas ("El Alumno" y "El instructor") se enmarcan dentro del interés técnico donde la educación tiene una función reproductora de la estructura social dominante (Chomsky, 2007; Grundy, 1998). La EF está orientada al desarrollo de la condición física y el dominio de determinadas habilidades motrices y la evaluación se plantea desde una perspectiva sumativa y calificativa (López-Pastor, 1999; López-Pastor, Monjas \& Pérez, 2003).

El interés práctico se refleja en la tercera etapa ("El Maestro"). Tanto alumnos como docentes son considerados sujetos activos del proceso educativo, que está centrado en el aprendizaje más que en la enseñanza (Grundy, 1998). Desde esta perspectiva, en el área de EF se busca el desarrollo integral del individuo mediante el cuerpo y la actividad física, utilizando la experimentación, la participación, la indagación y el descubrimiento. La evaluación se plantea desde una perspectiva continua y formativa, que busca ser útil para el alumnado a quien el docente va cediendo responsabilidades, empoderándolo y compartiendo con él este proceso (Álvarez-Méndez, 2001; Devís \& Molina, 2001; López-Pastor, 2006). 
En la última etapa ("El Educador") se aprecia su transcurrir hacia el interés emancipador. Desde éste se incita al alumnado a cuestionar y desafiar las creencias y prácticas represivas impuestas por la sociedad, y tiene como objetivo el desarrollo de la conciencia crítica y la capacidad del individuo para la transformación social a partir de valores como la justicia, la solidaridad, la igualdad y la dignidad (Flecha \& Villarejo, 2015; Freire, 1997; Giroux, 2001; Mclaren, 2005). Desde la perspectiva de la evaluación, ésta va evolucionando hacia lo que el protagonista define como la "Evaluación para el Bien Común", concepto que hemos aplicado a la educación y la evaluación a partir de la propuesta de Felber (2012), que lo utiliza desde el ámbito de la economía y el cambio del modelo social. En este sentido, esta evaluación utilizada en el ámbito de la EF pretende ir más allá de medir, valorar y aprender, ya que además promueve el compromiso con uno mismo y con la sociedad. Pretende favorecer el desarrollo emocional del alumno a la vez que las relaciones interpersonales y las dinámicas sociales entre los diferentes miembros de la comunidad educativa, estimulando el sentimiento de responsabilidad necesario para una implicación activa en la sociedad.

En cuanto a las implicaciones éticas del relato, se ha velado por realizar una escritura honesta, respetuosa, coherente y crítica (Sañudo, 2006) que permita aproximar al lector a la comprensión de las diferentes situaciones vitales de manera cercana y personal.

\section{RESULTADOS Y DISCUSIÓN}

Tal como hemos expuesto, los resultados se han explicado en torno a las cuatro fases de desarrollo profesional que surgen del relato autobiográfico. En cada una de estas fases hay dos partes: (a) la presentación del relato autobiográfico; (b) la discusión sobre dicho relato.

\subsection{EL ALUMNO}

Mi etapa como alumno ha sido uno de los periodos que más ha condicionado mi desarrollo personal. Durante la educación básica mi objetivo académico se centraba en conseguir acumular la mayor puntuación posible en las notas, para conseguir una buena media y acceder a estudiar una titulación universitaria donde dejar de hacer esas asignaturas obligatorias que tan poco me gustaban. Donde más disfrutaba era en EF, posiblemente porque podía correr y jugar, aunque las sesiones siempre tenían la misma estructura: una primera parte con ejercicios físicos y tablas gimnásticas, y una segunda donde se jugaba fútbol o baloncesto. En la última semana de cada trimestre se realizaba un test de resistencia o de habilidades, que servía para obtener las calificaciones. Se daban casos absurdos e injustos como el de un compañero que suspendió un trimestre por no saber hacer el pino y, después de pasarse meses practicándolo, la prueba de recuperación consistió en hacer el test de Cooper. En esa etapa obligatoria aprendí poco sobre el área de EF, pero al estar vinculado al deporte en diferentes actividades y clubs, las calificaciones iban siendo altas. Estas acostumbraban a ir acompañadas de frases como "supera muy bien los objetivos de la evaluación" (los cuales desconocía) o "tiene buenas cualidades para las actividades deportivas". El sistema de evaluación no medía lo que aprendía, sino lo que era. En cualquier caso, no recuerdo haber tenido interés en aprender temáticas académicas, pero sí recuerdo el esfuerzo y la dedicación en conseguir salvar un examen o sacar una nota para recibir el consiguiente reconocimiento. 
Empecé a estudiar Magisterio de EF y descubrí que tampoco sería un camino motivante hacia el aprendizaje. El plan docente volvía a estar orientado a mostrar al profesor que sabía repetir lo que él ya había dicho. Se volvían a dar sinsentidos, como cuando suspendí el examen final (y único) de una asignatura aun habiendo aprobado el trabajo final (y único). Creía haber sabido plasmar los apuntes tomados durante todo el semestre, pero el examen estaba suspenso. Finalmente, aunque el profesor me causaba mucho respeto, conseguí armarme de valor y presentarme en la revisión. El problema era matemático: al volver a hacer el sumatorio había un error de 4 puntos y la calificación superaba el 7 sobre 10. Por suerte ahorré dos meses más de estudio y quién sabe si una nueva matriculación. En otra ocasión, en una asignatura de iniciación deportiva la calificación final dependía al 50\% de una práctica. Este tenía tres pruebas de habilidad, en la que una consistía en realizar un circuito conduciendo una pelota de plástico con un stick del mismo material, sin tocar ningún cono y, al llegar a un punto señalado, golpearla para intentar meter gol en una pequeña portería situada a 6 metros. En función de si se lograba en el primer intento, en el segundo o en el tercero, se obtenía una calificación u otra. Una compañera, que en aquel momento jugaba en la selección nacional de hockey, falló los tres intentos. El profesor, al descubrir lo absurdo de la situación la aprobó, ya que "era jugadora de hockey". Sin embargo, dos compañeros más que tampoco lo lograron, suspendieron esa parte de la asignatura y tuvieron que volverla a realizar dos meses después, en segunda convocatoria. Si ese instrumento de evaluación era injusto por aleatorio para una persona, ¿no lo era también para el resto? Durante años los alumnos han continuado jugándose la nota en el mismo ejercicio. Un claro ejemplo de las incoherencias pedagógicas y educativas de un sistema de evaluación-calificación basado en las habilidades motrices.

Este sistema evaluativo llevaba a un modelo educativo jerárquico, donde el poder residía en el docente. Este calificaba, clasificaba y tomaba la última decisión sobre quién salvaba el muro y quién no. El rol del alumno era de obediencia y aceptación acrítica del orden establecido. El esfuerzo tenía valor en función de la nota obtenida. De hecho, cuando aprendía algo y estaba motivado, no acostumbraba a considerarlo un esfuerzo.

Muchas de las experiencias que muestra este relato sobre los absurdos sistemas de evaluación-calificación sufridos en la asignatura de EF, tanto en la educación obligatoria como en la formación inicial del profesorado (FIP), son las mismas que han vivido y siguen viviendo gran parte de los estudiantes españoles en las diferentes etapas educativas. Este injusto sistema de evaluación-calificación ha recibido numerosas críticas en las dos últimas décadas (Córdoba, 2015; López-Pastor, 1999, 2006, 2013; Méndez, 2005), pero sigue reproduciéndose, generación tras generación. Se le suele definir como "sistema tradicional de evaluación-calificación en EF" (López-Pastor, 1999, 2006). Se basa de manera prioritaria en la medida de la condición física y motriz del individuo, utilizando la realización de test de condición física y de habilidad motriz para clasificar y penalizar al alumnado. Las críticas a este sistema se centran en su falta de valor educativo, la absurda e injusta lógica en que se basan y las grandes incoherencias internas que supone respecto a los objetivos y procesos de aprendizaje.

Es un modelo que entiende la evaluación como forma de control y poder disciplinario del estudiante y del grupo clase (Álvarez-Méndez, 2001; López-Pastor, 1999; Santos-Guerra, 2003). En esta forma de racionalidad técnica el alumno es un actor pasivo del proceso educativo, que aprende a partir de objetivos prescriptivos cuya implementación dependerá de la habilidad del profesor, el actor principal. Promueve una función reproductora de la 
sociedad que pretende mantener la estructura social (Chomsky, 2007). Los sistemas de evaluación utilizados más frecuentemente están orientados de forma exclusiva y obsesiva a la calificación, destrozando el deseo de aprender y limitando el proceso educativo a ir superando obstáculos y niveles, y a un intercambio de trabajos y tiempo de estudio por notas (Álvarez-Méndez, 2001; Santos-Guerra, 2015). El aprendizaje estará determinado por los sistemas de evaluación utilizados, y este podrá ser tan bueno como lo sean las tareas de evaluación que se propongan (Pérez \& González, 2011; Santos-Guerra, 2015). La estructura de sesión que se menciona en la primera etapa ha sido bastante habitual en España en la década de los ochenta y los noventa (Córdoba, 2015; López Pastor et al., 2005). Se alternaban sesiones más centradas en entrenamientos físicos, basados en la estructura de calentamiento, parte principal y vuelta a la calma, con sesiones completas dedicadas a "echar partidillos" o "deporte libre" o bien, como se ven en este caso, una mezcla de ambas tendencias. Esta alternancia es una de las causas que han repercutido en la pérdida de estatus de la EF (Barba, 2011; González \& Barba, 2013; López-Pastor et al., 2005; Pascual, 2003; Pérez-Pueyo, 2005).

\subsection{EL INSTRUCTOR}

Al concluir la FIP y cambiar del rol de alumno al de docente también cambió la perspectiva que tenía de muchas situaciones educativas, aunque mi paradigma educativo seguía anclado en mi etapa anterior. Pasé de dominado a dominante, utilizando de manera inconsciente ese perfil jerárquico, autoritario y reproductor del orden social que había sufrido con anterioridad. Abusaba del poder que me otorgaba la evaluación, siendo ésta una herramienta que utilizaba para menesteres varios: reafirmar mi poder ("si alguien habla cuando yo explico le bajaré la nota"), premiar ("quien me ayude a recoger el material tendrá un positivo"), clasificar ("los cinco primeros que lo consigan tendrán esta nota, los cinco siguientes esta otra.... y los cinco últimos deberán repetirlo en la siguiente sesión”), y castigar ("si te sigues portando mal y no respetas a tus compañeros suspenderás este trimestre"). No me planteaba que las dificultades que se daban en los procesos de aprendizaje y las calificaciones bajas pudieran deberse a un sistema de evaluación incoherente o a mi mala praxis docente. La responsabilidad de haber aprobado o suspendido la atribuía siempre al alumno. Aunque pareciera lo contrario, no había ninguna mala intención en esa actuación, y en mi ánimo estaba el tener buena relación con los alumnos y ofrecerles el máximo de aprendizajes posibles.

Definiría mi perfil docente como el de un instructor. Mandaba una serie de tareas y actividades, a poder ser lúdicas, para que los alumnos aprendieran y reprodujeran aquello que consideraba necesario para aprobar la asignatura. La evaluación tenía un objetivo diferente en función de quien la interpretara: para mí era seleccionar, seguir poniendo obstáculos, mientras que para los alumnos era evitar el fracaso y conseguir superarlos. Aunque parezca grotesco, poco se diferenciaba mi función de la de un adiestrador canino. En las sesiones había circuitos, dominio de técnicas y habilidades, juegos dirigidos y recompensas y sanciones con un doble fin: el desarrollo motriz y la obediencia.

Era asombroso el poder que me otorgaba una libreta con el listado de alumnos cuando, durante las sesiones, paseaba con ella y un bolígrafo. Intentaba anotar todo lo que pasaba en cada clase mediante números, signos positivos o negativos y algún que otro comentario, para poder utilizarlo al final de trimestre y rellenar de la manera más "objetiva" posible 
los informes, encontrándome siempre con los mismos problemas: lo anotado poco se correspondía con los ítems que aparecían en los informes; la mayoría de estas anotaciones eran siempre en negativo (se ha peleado, no ha conseguido hacer la voltereta, no aguanta el equilibrio...), debiendo interpretar que aquellos alumnos que no tenían anotaciones debían hacer bien las cosas; y que el ítem más utilizado era el referente a la higiene (ducha y cambio de ropa). De hecho, éste era el único aspecto sobre el que se tomaba nota en todas las sesiones, teniendo un peso importante en la calificación final.

Yo "hacía de profesor" tal cual lo había vivido como alumno, sin llegar a cuestionarme mi práctica o concepción educativa. Era "lo que se había hecho siempre” y, por lo tanto, lo que se debería seguir haciendo, aunque era incompatible con mi manera de ser, sentir y vivir. Por esa razón empecé a estudiar Ciencias de la Actividad Física y el Deporte (CAFD), confiando en poder dejar la docencia y dedicarme a otras tareas profesionales vinculadas al deporte.

Como puede comprobarse, los absurdos sistemas de evaluación-calificación sufridos en EF durante la educación obligatoria también parecen reproducirse durante la FIP durante esas décadas (López-Pastor, Pérez \& García-Peñuela, 2001). Si su utilización en primaria y secundaria se considera anacrónica, poco educativa, no orientada al aprendizaje e injusta, mantenerla en FIP genera críticas aún más graves: incoherente, absurda, sin sentido y generadora de malas praxis profesionales. La evaluación de un profesional de la EF debería ser sobre sus competencias docentes, no sobre su forma física o su habilidad deportiva. Según Flores y Day (2006), la construcción de la identidad profesional docente está influenciada por tres factores determinantes: las experiencias previas, la formación inicial y el impacto del contexto de enseñanza. Esto ha supuesto un problema en la innovación y el progreso del sistema educativo, ya que ha favorecido la reproducción de las ideas educativas preconcebidas y los modelos de enseñanza tradicionales que han conformado la experiencia educativa vivida de gran parte del colectivo docente. Muchas de las experiencias vividas por el docente de EF durante su paso como alumno por la escuela, y posteriormente en su FIP, determinan en gran parte las características y enfoques de su práctica docente (Córdoba, 2015).

La evaluación que utiliza el protagonista en esta etapa se enmarca en el aprendizaje bancario, transmitiendo contenidos fijos que no requieren de reelaboración, donde el docente otorga y el alumno recibe (Freire, 1997). La calificación es utilizada como herramienta de poder, para controlar al alumnado a partir de penalizaciones (Álvarez-Méndez, 2001; Santos-Guerra, 2003), manifestándose como un acto violento considerando al otro como un ignorante que debe aprender (Freire, 1997). Partiendo de Santos-Guerra (2003) y Murillo e Hidalgo (2015) se puede secuenciar esta manera de evaluar a partir de tres pasos básicos: (a) "comprobación": "el que sabe" le dice "al que no sabe" que ha hecho bien o mal según su propio criterio; (b) "clasificación": se aplicaban categorías a los alumnos donde se magnifican sus áreas débiles y el autoconcepto negativo, cumpliendo las profecías del docente y estableciendo una jerarquía social dentro del grupo; y, (c) "atribución": como el docente ha enseñado, él alumno debe haber aprendido, y si no lo ha hecho es por su falta de responsabilidad.

La concepción de EF del protagonista se enmarca en esta fase en una estructura mecanicista orientada a conseguir objetivos de rendimiento mesurables (Velázquez \& Hernández, 2005). Entiende el cuerpo como una máquina y se basa en un modelo conductista, repitiendo estímulos y midiendo el rendimiento resultante. Es una EF de racionalidad 
técnica, orientada al rendimiento físico-deportivo y enfocada al desarrollo de la condición física mediante el adiestramiento físico y el dominio de las habilidades físico-deportivas (López-Pastor, 1999, 2006). La evaluación-calificación se basa en la aplicación sistemática de test de condición física y/o habilidad motriz. Esta ha sido ampliamente criticada en la bibliografía de las tres últimas décadas (Gipps \& Murphy, 1994; López-Pastor, 1999, 2006; Méndez, 2005) por la incoherencia, la superficialidad y el reduccionismo respecto al área de EF, por no estar orientada al aprendizaje, sino principalmente a la calificación y selección.

\subsection{EL MAESTRO}

Estudiando CAFD y cursando asignaturas de pedagogía y didáctica de la EF, retomé la preocupación por mi práctica y desarrollo docente. La inflexión en mi perfil profesional fue en el Congreso de Actividades Físicas Cooperativas, en el año 2003, en Gijón, donde asistí para presentar un trabajo universitario. Empecé a identificar aquellas prácticas que no deseaba volver a reproducir y aposté por un modelo cimentado en las dos ideas fundamentales que me había llevado del congreso: (a) mediante las metodologías cooperativas se aprende más y mejor; y, (b) la evaluación formativa es parte del proceso de aprendizaje y debe servir para formar, aprender y dar autonomía al alumnado. Al volver del congreso tenía una imperiosa necesidad de seguir aprendiendo sobre sistemas de evaluación formativa y compartida (EFyC). Intuía que estas ideas podrían ser el pilar de esa tarea docente que ansiaba realizar y que estaban en sintonía con lo que deseaba que fuera la educación. No recordaba haberlas tratado de manera específica en mi FIP. Al revisar apuntes de los años de estudio encontraba citas a la evaluación inicial, continua y sumativa, pero de EFyC nada. Me decidí a buscar formaciones, pero la oferta de cursos donde la EFyC fuera un contenido importante era inexistente. Durante algunos años el recurso al que tuve acceso fue la bibliografía, tanto desde una perspectiva general como específica de EF y tomando como referencias publicaciones de Álvarez-Méndez (2001), López-Pastor (2006), Sanmartí (2007) y Santos-Guerra (2003). Todo ello lo compartía con compañeros de Grupos de Trabajo de formación permanente de EF (GT de Mestres d'EF de Cerdanyola, Grup de Formació de Formadors de l'ICE de la UAB), con inquietudes parecidas, donde construíamos y reconstruíamos conjuntamente nuestros saberes docentes relativos a la evaluación, entre otros temas.

La asistencia al III Congreso Nacional de Evaluación Formativa en la Universidad, celebrado en Barcelona en 2008, supuso otro gran paso en mi particular inmersión en EFyC. Descubrir a tantos profesionales de la educación preocupados por esta evaluación como elemento crítico del proceso de aprendizaje resultó un estímulo extraordinario en mi práctica docente. Ese modelo de evaluación generó un cambio radical en mi manera de entender al alumno. Del individuo con unas capacidades innatas e inamovibles que lo condicionan al éxito o al fracaso, al individuo competente para desarrollar las capacidades que desee y ser protagonista en su desarrollo personal. Así, de un instructor preocupado en cómo enseñar, fui convirtiéndome en un maestro preocupado en cómo se aprendía.

La combinación de EFyC con metodologías cooperativas para el aprendizaje resultó un cóctel exitoso. No era lo mismo decir a los alumnos que para aprobar cada uno de ellos debía saber saltar la cuerda de cuatro maneras diferentes, que proponerles un reto donde todos los miembros de un grupo debían conseguir saltar la cuerda como mínimo 
de cuatro maneras diferentes, siendo ellos mismos quienes controlarían y gestionarían ese proceso. Otro ejemplo se daba al utilizar un marcador colectivo de modo que los logros de cada alumno (número de encestes, número de vueltas sin dejar de correr, número de metros saltados...) sumaban en el marcador de toda la clase y permitían alcanzar un reto previamente propuesto. El hecho de crear esa interdependencia favorecía las relaciones entre el alumnado, se celebraban los éxitos propios y ajenos, aumentaba la motivación, provocaba la autorregulación del aprendizaje y los logros se alcanzaban con más facilidad. Se disipaba el enseñar y se magnificaba el aprender, siendo los propios alumnos quienes se responsabilizaban del propio proceso como del de los compañeros.

El elemento determinante en la consolidación de EFyC fue ser capaz de desvincular la evaluación de la calificación. Los alumnos asociaban la calificación a recompensas, reprimendas y a un estatus social dentro del grupo, siendo el objetivo de su esfuerzo aprobar o alcanzar una nota. Quería utilizar un sistema de evaluación basado en la honestidad y la confianza, que realmente sirviera para aprender, donde los aprendizajes despertaran interés en el alumnado, fueran relevantes y los consideraran de utilidad.

Empecé a utilizar actividades de evaluación durante las sesiones de EF con la finalidad de que cada alumno pudiese ir valorando y recibiendo feedback constante de sus compañeros y mío sobre lo que aprendía y la manera en que lo hacía. Así, era muy bueno que un alumno hiciera cosas bien y se valorara positivamente; pero también era muy bueno que fuera consciente de aquello que no le salía tan bien e intentara mejorarlo (autoevaluación). En el mismo sentido, era muy enriquecedor ver como entre los alumnos se daban feedback positivo ante los logros conseguidos, pero todavía lo era más cuando, desde el respeto, eran capaces de corregirse y expresar sus descontentos u opiniones no tan positivas, pretendiendo que un compañero mejorara algún aspecto (coevaluación). Mi identidad docente empezaba a ser coherente con el rol del adulto que también comparte su experiencia y que contribuye, con sus opiniones y puntos de vista, a que el alumno aprenda más, y no como "el que todo lo sabe" y debe trasmitirlo. De este modo, el desarrollo de mis unidades didácticas fue tomando un mismo patrón: (1) se organizaban equipos de trabajo de 4 o 5 personas que acostumbraban a durar un trimestre y, por lo tanto, trabajaban conjuntamente en 2 o 3 unidades didácticas. En función del número de participantes que requería cada actividad, se establecían 2 o más grupos, facilitando la relación con el resto de compañeros; (2) se proponía a los grupos unos retos que partían de los objetivos didácticos diseñados para esa unidad. Se exponían los niveles mínimos de consecución que todos los miembros del grupo debían lograr, y se dejaban abiertos los máximos. Cada equipo podía reformular el reto a conseguir y el nivel a alcanzar, consensuándolo conmigo. Un grupo no lograba el éxito hasta que todos y cada uno de sus miembros no hubiera dado evidencias de la consecución del nivel mínimo acordado; (3) durante las sesiones, los equipos trabajaban de manera autónoma o de forma guiada. Disponían de una hoja de seguimiento donde podían ir apuntando información respecto al grado de consecución de cada uno de los retos. También podían apoyar a otros equipos si así lo consideraban; (4) al final de cada sesión cada grupo se reunía durante 5 minutos para poner en común lo sucedido y anotar aquello relevante respecto a lo pretendido; (5) si en las sesiones previstas algún grupo no había conseguido los retos mínimos, tenía oportunidad de dedicar un pequeño espacio de tiempo en sesiones posteriores a practicar y exponer las evidencias de consecución.

¿Y la calificación? Intentaba desvincularla del proceso de aprendizaje. Como "poner notas" resultaba un imperativo legal al final de cada trimestre, opté por utilizar el método 
de la calificación dialogada (López-Pastor, 2006). La última semana antes de la junta de evaluación pedía a cada alumno que valorara su participación en la asignatura durante el trimestre, en función de los objetivos que se habían propuesto, utilizando las hojas de seguimiento individual y grupal utilizadas durante el trimestre. Al final de esta valoración debían sugerir una autocalificación que consideraran justa y honesta. Yo también realizaba el mismo proceso y hacía una valoración de cada alumno partiendo de lo anotado en el cuaderno del profesor. Cabe señalar que la utilidad fundamental de dicho cuaderno era ir anotando información relativa al proceso de aprendizaje, para dar un feedback lo más inmediato posible. Además, al final de cada trimestre también lo utilizaba para construir una opinión general del proceso de cada alumno. En las dos sesiones posteriores, mientras el gran grupo desarrollaba sesiones de carácter autónomo que no requirieran de mi participación, yo efectuaba una pequeña entrevista con cada alumno, donde compartíamos esas valoraciones. Si la calificación coincidía, la manteníamos; en el caso de que fuera diferente, dialogábamos sobre los criterios que manejábamos y acordábamos una calificación a partir del principio de honestidad. Finalmente, la sensación que nos llevábamos tanto alumnos como yo era la de un proceso justo y ético, donde el valor prioritario era el de aprender y no el de aprobar.

Margalef (2014) considera que para el profesorado, implementar EFyC ha supuesto superar resistencias y paradojas como las concepciones y creencias previas sobre enseñanza, aprendizaje y evaluación; la cultura organizativa y las condiciones institucionales; la incertidumbre e inseguridad que generan los procesos de cambio; y los escasos referentes en evaluación formativa en los que apoyarse. Algunos estudios recientes empiezan a indicar que existe cierta transferencia entre la experimentación y vivencia como alumnos de sistemas de EFyC durante la FIP y su posterior aplicación en la práctica docente (Hamodi, LópezPastor \& López-Pastor, 2017; Palacios \& López-Pastor, 2013). Sin embargo, en este relato la transformación profesional desde sistemas de evaluación-calificación tradicional hacia la EFyC surge fundamentalmente a partir de la formación permanente y de los procesos reflexivos que ésta genera (Molina \& López-Pastor, 2017). Esos procesos de formación dialógicos y colectivos en los que el debate y el diálogo con otros iguales son los que ayudan a cuestionarse las propias convicciones y a avanzar hacia nuevos planteamientos. Pueden encontrarse ejemplos y relatos de estos procesos de desarrollo profesional colectivo en: Barba-Martín y López-Pastor (2017), Blández (1996), Córdoba (2015), Córdoba et al. (2016), González y Barba (2014), Hamodi, López-Pastor y López-Pastor (2014) y LópezPastor et al. (2016). Muchos de estos autores conceden gran importancia a actividades de formación permanente como (a) cursos, jornadas y congresos, especialmente aquellos en que se debaten e intercambian ideas y experiencias prácticas; (b) espirales, ciclos y procesos de investigación-acción sobre su práctica educativa; (c) seminarios, fórums o grupos de trabajo de profesores de $\mathrm{EF}$, donde se ponen en común experiencias, dudas y respuestas diversas en torno a una línea de trabajo, ligados, en algunos casos, a ciclos de investigación-acción.

En el relato se incide especialmente en la necesidad de lograr una fuerte coherencia entre las metodologías cooperativas y los sistemas de evaluación basados en la autorregulación del aprendizaje por parte del alumnado, tal como defienden numerosos estudios (Fernández-Rio, 2003; Johnson, Johnson \& Holoubec, 1999; López-Pastor et al., 2008; Pujolás, 2008; Velázquez-Callado, 2010). Según estos planteamientos, una evaluación coherente con el aprendizaje cooperativo debería caracterizarse por (a) estar integrada en 
el proceso de aprendizaje grupal y cooperativo; (b) desarrollar procesos de autoevaluación y coevaluación; (c) utilizar instrumentos de evaluación variados, y (d) valorar tanto logros motores como cognitivos, sociales y actitudinales.

Aunque la implementación de metodologías cooperativas en EF en las últimas décadas ha crecido considerablemente, no ha sido hasta hace pocos años que han aparecido documentos que acompañan al docente en esta puesta en práctica y que ofrecen estructuras estables que permiten comprender, utilizar e integrar el aprendizaje cooperativo en EF desde situaciones sencillas a complejas (Córdoba \& Romero, 2016; Fernández-Río, 2017, Ruíz-Omeñaca, 2017; Velázquez-Callado, 2010).

En los últimos 25 años se han publicado algunas experiencias sobre la utilización de sistemas de autocalificación y calificación dialogada en EF (Castanedo \& Capllonch, 2017; Herranz, 2013; López-Pastor, 1999, 2006; López-Pastor \& Pérez-Pueyo, 2017; Pérez-Pueyo, 2015). Muchos de ellos han sido llevados a cabo en primaria. Los estudios realizados muestran que se trata de procedimientos fiables y eficaces, obteniendo resultados muy positivos en la mejora del clima de aula, el aprendizaje, el rendimiento académico y el comportamiento del alumnado.

\subsection{EL EDUCADOR}

En los cursos posteriores, la reflexión permanente a partir de la puesta en práctica de metodologías cooperativas y sistemas de evaluación formativa me permitió cuestionarme muchos elementos de mi tarea docente. Modificaba técnicas y estrategias buscando consolidar una práctica al servicio del aprendizaje de los alumnos. Este hecho, junto a la inmersión en la pedagogía crítica, despertó una preocupación cada vez más importante por la transferencia del hecho educativo a la sociedad.

Aunque utilizar metodologías cooperativas favorecía el florecimiento de los principios básicos de la relación humana, mi práctica continuaba centrada en la dimensión técnica del aprendizaje: en el éxito académico del alumnado y en su desarrollo motriz e intelectual. Corría el riesgo, tan habitual en EF, de que alumnos con un gran dominio de habilidades propias del área, amparándose en ellas, generaban situaciones no deseadas: ensalzamiento del componente agonístico de las actividades, no asunción de responsabilidades, búsqueda de estatus personal a costa de los derechos de los demás, generación de conductas disruptivas, actitudes irrespetuosas con los menos dotados motrizmente o falta de respeto a las normas consensuadas. Aunque me esforzaba en el desarrollo del alumno, sentía no prestar atención al compromiso social.

Estas inquietudes se magnificaron con el impacto en mi entorno social de la crisis económica en 2008. Lecturas a priori alejadas del tema educativo que proponían un modelo económico basado en la cooperación y el bien común (Felber, 2012) fueron determinantes para fundamentar mi práctica educativa desde la vertiente social y política. Además, fue clave el cursar un Máster sobre Educación Inclusiva, abriéndome la óptica de una educación para todos, respetuosa con las diferencias al servicio de la sociedad y a su transformación en base a la justicia social. Entendí que el objetivo de la institución escolar, y el mío como miembro de ésta, debía ser ofrecer un contexto en el que se aprendiera a sentir amor por el otro, por uno mismo, por la familia, por el entorno y por la cultura. Donde se aprendiera a pensar de manera crítica, actuando éticamente, conviviendo digna y empáticamente, y afrontando con liderazgo, cooperación y autonomía los retos personales y sociales. Un 
modelo educativo basado en el bien común edificado sobre cuatro preguntas claves: (1) ¿Qué tipo de persona debe formar la escuela?; (2) ¿Qué tipo de sociedad debe construir esa persona?; (3) si ese es el tipo de persona y sociedad que debe construir la escuela, ¿Cuál es el modelo educativo que se necesita?; y entonces, (4) ¿Qué tipo de sistema de evaluación necesita este modelo educativo? Es un modelo que parte de los cuatro pilares del aprendizaje de Delors (1996), pero donde el aprender a conocer y el aprender a hacer quedan supeditados al aprender a ser y al aprender a vivir juntos. No se deja de lado la valoración de la adquisición de competencias académicas, científicas, motrices, cognitivas, emocionales, tecnológicas, de liderazgo y de emprendimiento, pero sí que las condiciona a la adquisición paralela de otras competencias imprescindibles en una sociedad del bien común, como las éticas, las morales, las afectivas y las sociales y ciudadanas.

La coherencia de este modelo pedagógico con la manera que tenía de ver, sentir e interpretar la vida me dio ilusiones renovadas para implementarlo en mi práctica. Entendía que la EF, como el resto de áreas, tenía como finalidad última contribuir a la adquisición de las competencias anteriormente nombradas, pero que a la vez se apoyaba en las tres herramientas fundamentales para el aprendizaje: el cuerpo, el movimiento y el juego. Estaba convencido de que era bastante improbable ser capaz de aprender sentado en una silla, quieto y mirando una pizarra, ya que seguramente nuestra historia evolutiva no nos había preparado genéticamente para ello.

Empecé a utilizar instrumentos en la evaluación que no se limitaran a valorar las tareas realizadas en el contexto de las sesiones de EF, sino que pretendieran extrapolarlas a la vida cotidiana. En las hojas de seguimiento del alumnado de cada unidad didáctica fue cobrando cada vez más importancia la aplicación de los aprendizajes y aportación de evidencias en contextos diferentes a la sesión de EF. Por ejemplo, en una unidad didáctica donde se utilizaba el fútbol para contribuir a las competencias de aprender a aprender y social y ciudadana tenían que mostrar evidencias donde hubieran sido capaces de aprender y/o enseñar técnicas y habilidades del fútbol a otras personas, y sí habían tenido actitudes éticas, solidarias y honestas cuando lo practicaban, tanto en clase como en otros entornos diferentes (patio, parque, familia...). Estas evidencias se mostraban a partir de respuestas a las siguientes preguntas: “ ¿Has sido capaz de aplicar lo aprendido en otros lugares? ¿Ha sido de ayuda para otras personas?”, “¿Has sido capaz de disfrutar y de ayudar a que el resto también disfrute de la sesión?”, o “¿Te comprometes a llevar a cabo una acción con tu familia o con tus amigos relacionada con lo aprendido?". La pretensión era conseguir que lo aprendido fuera significativo y relevante también en el entorno social del alumno. Para ello era imprescindible una "Evaluación para el Bien Común", donde las técnicas e instrumentos de evaluación se aplicaran a situaciones que fueran más allá del progreso individual, buscando el logro y el beneficio social, y enfatizando valores imprescindibles para la convivencia social como la empatía, la amistad, la solidaridad, el espíritu crítico, la sinceridad, la responsabilidad y el respeto.

Es así como las diferentes perspectivas de la evaluación han contribuido a hacer evolucionar el perfil profesional de este docente. Un perfil sustentado sobre tres principios fundamentales que le han permitido dar rigor y solidez al proceso de educar: (1) la ciencia, ya que se formula preguntas e investiga, pero responde con rigor y evidencias, y no con suposiciones y ocurrencias; (2) la consciencia, ya que se preocupa por las particularidades y necesidades de cada alumno, conociendo las diferentes realidades sociales y cumpliendo con las exigencias éticas, sociales y profesionales que conlleva su tarea; (3) el corazón, ya 
que es sensible al éxito, a la alegría, al dolor y al sufrimiento ajeno; (4) el coraje, ya que no ceja en el empeño y se compromete con el desarrollo de sus alumnos y de la sociedad.

Dada la crisis sistémica creciente que estamos viviendo, es urgente la necesidad de compromiso educativo hacia el desarrollo social y sostenible. Transformar una educación al servicio del mercado donde prima el individualismo, la competitividad, la rivalidad y el sacrificio, a una educación para el bien común con valores como la solidaridad, la empatía, la ayuda mutua y la responsabilidad compartida (Aznar \& Barrón, 2017; Entrena \& Martínez-Rodríguez, 2013; Rifkin, 2010; Torres, 2007).

Una visión humanista de la educación basada en el respeto a la vida y a la dignidad humana, la igualdad de oportunidades, la justicia social, la diversidad cultural, la solidaridad y la sostenibilidad (UNESCO, 2015) dota al hecho de educar de un incuestionable e importante valor político (Freire, 1997; Giroux, 2001; Mclaren, 2005). Como elemento curricular básico en el proceso educativo, la evaluación también es una actividad de marcado carácter político. El concepto de "bien común" aparece en el relato inspirado en el ensayo de Felber (2012) sobre un nuevo modelo económico en el que a partir de valores como la cooperación, la solidaridad y la necesidad de compartir, vincula lo económico y lo social. El nexo sobre el que se articula este cambio de mentalidad social es la educación y, por tanto, la utilización de sistemas de evaluación culturalmente sensibles, justos, participativos, que empoderen al individuo, y donde, además de valorar aspectos teóricos y procedimentales, sobre todo evalúen el autoconcepto de cada estudiante y las repercusiones sociales del aprendizaje (Murillo \& Hidalgo, 2015). Finalmente, los principios fundamentales de la profesión docente (ciencia, consciencia, corazón y coraje) están inspirados en la metáfora de Gervas (2012) sobre los elementos imprescindibles que debe llevar todo profesional sanitario en su "cabás".

\section{CONCLUSIONES}

Este trabajo sirve para mostrar la importancia que ha tenido la forma de entender la evaluación en el desarrollo personal y profesional de un docente concreto, por lo que no puede ni debe extrapolarse a cualquier realidad. Vamos a ir revisando en qué medida los datos presentados contestan a los tres objetivos del estudio.

El artículo muestra cómo los procesos de crecimiento profesional y personal del profesorado están fuertemente mediatizados por sus experiencias previas como alumnos de primaria y secundaria, así como por las experiencias acumuladas en su FIP, pero que también lo pueden estar por los procesos reflexivos que se desarrollen a lo largo de su vida profesional, mediante dinámicas de formación permanente y de investigación-acción. Estos procesos reflexivos suelen centrarse en elementos curriculares concretos, como han sido, en este caso, los sistemas de evaluación formativa y compartida y la utilización de metodologías cooperativas en EF. Este estudio muestra una evolución desde modelos muy tradicionales, basados en un interés constitutivo del saber de carácter "técnico", hacia modelos más innovadores, guiados por interés constitutivo de carácter "práctico" y, posteriormente, emancipatorio.

Se aprecia la manera en que la transformación del concepto de evaluación del protagonista, a lo largo de las diferentes etapas, ha sido simultánea y sinérgica con el cambio en su manera de sentir, interpretar y actuar en el contexto escolar. De estos procesos 
emerge un docente más preocupado en el aprendizaje de los alumnos que en la enseñanza, que promueve el empoderamiento del estudiante, que utiliza la evaluación formativa como herramienta fundamental para el proceso para aprender, que dialoga y acuerda la calificación con el estudiante, y que se muestra especialmente comprometido en la transformación de la sociedad hacia otra más justa y solidaria.

Los factores que más han influido en el desarrollo de sistemas de evaluación formativa por parte del docente han sido aquellos relacionados con la formación permanente: (a) el trabajo colectivo y cooperativo con otros docentes, compartiendo experiencias, inquietudes e ideas en grupos de trabajo, fórums y encuentros de profesorado; (b) la investigación bibliográfica; (c) la investigación-acción a partir de la propia práctica, y (d) la asistencia a jornadas y congresos relativos a la temática.

La utilidad de este relato autobiográfico puede extenderse a docentes que se encuentren en momentos vitales o profesionales similares a los descritos y les permita observar una manera de afrontarlos. También puede resultar interesante a investigadores de prácticas educativas, ya que expone un recorrido personal por el sistema educativo, partiendo del rol de alumno hasta el de docente consolidado, con todas sus reflexiones, inseguridades y certezas de lo que supone la educación desde la perspectiva de la evaluación. Por último, puede ser útil al alumnado de FIP, ya que analiza diferentes enfoques de la evaluación, uno de los aspectos que más controversias genera en el sistema educativo, y propone un modelo basado en el bien común, que sin duda será uno de los conceptos claves del siglo XXI.

Por último, este estudio genera unas prospectivas de futuro: (a) profundizar sobre las razones que puede tener el profesorado de EF para poner en práctica sistemas de EFyC con muestras más amplias, a partir de grupos focales y entrevistas en profundidad, y (b) contrastar el impacto de la evaluación para el bien común tanto en el contexto escolar como en el social.

\section{REFERENCIAS BIBLIOGRÁFICAS}

Álvarez-Méndez, J. M. (2001). Evaluar para conocer, examinar para excluir. Madrid: Morata.

Aznar, P., \& Barrón, A. (2017). El desarrollo humano sostenible: un compromiso educativo. Teoría de la Educación; Revista Interuniversitaria, 29, 25-53. doi:10.14201/teoredu20172912553

Barba, J. J. (2011). El desarrollo profesional de un maestro novel en la escuela rural desde una perspectiva crítica (Tesis Doctoral). Universidad de Valladolid, Segovia. Recuperado de http:// uvadoc.uva.es/handle/10324/822

Barba, J. J., González, G., \& Barba-Martín, R. A. (2014). Qué la fuerza esté contigo: Desvelar el lado oscuro de la investigación en educación. Magis: Revista Internacional de Investigación en Educación, 7(14), 125-140. doi:10.11144/Javeriana.M7-14.QLFE

Barba-Martín, R., \& López-Pastor, V.M. (2017). La transformación de los procesos de evaluación en educación infantil mediante la formación permanente a través de la investigación-acción. Infancia, Educación y Aprendizaje, 3(2), 674-679. doi:10.22370/ieya.2017.3.2.800

Barnett, R., \& Parry, G. (2014). Policy Analysis Research in Higher Education: Negotiating Dilemmas. Magis, Revista Internacional de Investigación en Educación, 7(14), 69-84. doi:10.11144/ Javeriana.M7-14.PARH

Blández, J. (1996). La investigación-acción: un reto para el profesorado. Guía práctica para grupos de trabajo, seminarios y equipos de investigación. Zaragoza: INDE.

Blázquez, D. (2017). Cómo evaluar bien en educación física. El enfoque de la evaluación formativa. 
Barcelona: INDE.

Blázquez, D., \& Sebastiani, E.M. (2016). ¿Cómo se evalúan las competencias? En T. Lleixa, y E. M. Sebastiani (Coord.), Competencias clave y Educación física. ¿Cómo y para qué tenerlas en cuenta en la programación? (pp. 109-126). Barcelona: INDE.

Bolívar, A., Domingo, J., \& Fernández, M. (2001). La investigación biográfico-narrativa en educación. Enfoque y metodología. Madrid: La Muralla.

Bonsón, M., \& Benito, A. (2005). Evaluación y Aprendizaje. En A. Benito y A. Cruz (Coords.), Nuevas claves para la docencia universitaria en el Espacio Europeo de Educación Superior (pp. 87-100). Madrid: Narcea.

Brown, S., \& Glasner, A. (2003). Evaluar en la universidad. Problemas y nuevos enfoques. Madrid: Narcea.

Castanedo, J.M., \& Capllonch, M. (2017). La Evaluación Formativa y Compartida en el Modelo Inclusivo Retos Individuales con Responsabilidad Compartida. Revista Infancia, Educación y Aprendizaje, 3(2), 118-126. doi:10.22370/ieya.2017.3.2.710

Chomsky, N. (2007). La (des)educación. Barcelona: Crítica.

Córdoba, T. (2015). La aventura de aprender: Relato autobiográfico del viaje a Ítaca de un docente reflexivo. RETOS. Nuevas tendencias en educación física, deporte y recreación, 28, 285-290. Recuperado de http://www.redalyc.org/html/3457/345741428049/

Córdoba, T., Carbonero, L., Sánchez, D., Inglada, S., Serra, M., Blasco, M., . . Sáez, S. (2016). Educación Física Cooperativa, formación permanente y desarrollo profesional. De la escritura colectiva a un relato de vida compartido. RETOS. Nuevas tendencias en educación física, deporte y recreación, 29, 264-269. Recuperado de http://www.redalyc.org/html/3457/345743464049/

Córdoba, T., \& Romero, R. (2016). Cooperar en l'activitat física i l'esport. 50 propostes pràctiques. Barcelona: Institut Barcelona Esports-Ajuntament de Barcelona.

Delors, J. (Coord). (1996). La educación encierra un tesoro. Informe a la UNESCO de la Comisión Internacional sobre la Educación para el Siglo XXI. París: UNESCO.

Devís, J., \& Molina, J. P. (2001). Los estudios del currículum y la Educación Física. En B. Vázquez (Ed.), Bases educativas de la actividad física y el deporte (pp. 243-276). Madrid: Síntesis.

Entrena, S., \& Martínez-Rodríguez, F. M. (2013). Educación y cambio social: del capitalismo neoliberal a la economía del bien común. En S. Torío, O. García-Pérez, J.V. Peña y C.M. Fernández (Coords.), Crisis social y el estado del bienestar: las respuestas de la pedagogía social (pp. 72-77). Oviedo: Universidad de Oviedo.

Felber, C. (2012). La economía del bien común. Barcelona: Deusto.

Fernández-Río, J. (2003). El aprendizaje cooperativo en el aula de educación física para la integración en el medio social: análisis comparativo con otros sistemas de enseñanza y aprendizaje. [CDROM]. Valladolid: La Peonza.

Fernández-Rio, J. (2017). El Ciclo del Aprendizaje Cooperativo: una guía para implementar de manera efectiva el aprendizaje cooperativo en educación física. RETOS. Nuevas tendencias en educación física, deporte y recreación, 32, 264-269. Recuperado de https://recyt.fecyt.es/index. $\mathrm{php} / \mathrm{retos} / \mathrm{article} / \mathrm{view} / 51298$

Flecha, R., \& Villarejo, B. (2015). Pedagogía Crítica: Un Acercamiento al Derecho Real de la Educación. Revista Internacional de Educación para la Justicia Social, 4(2), 87-100. doi:10.15366/riejs2015.4.2

Flores, M. A., \& Day, C. (2006). Contexts which shape and reshape new teacher's identities: A multi-perspective study. Teaching and Teacher Education, 22(2), 219-232. doi:10.1016/j. tate.2005.09.002

Freire, P. (1997). Pedagogía de la autonomía. Saberes necesarios para la práctica educativa. Madrid: Siglo XXI.

Gérvas, J. (2012). El cabás y el profesional sanitario. Cuatro maletines que definen el trabajo. Gaceta Médica de Bilbao, 109(3), 89-92. 
Gipps, C., \& Murphy, P. (1994). A fair test? Assessment, achievement and equity. Bristol: Open University Press. Recuperado el 23 de abril de 2017 desde http://psycnet.apa.org/ record/1996-97311-000

Giroux, H. A. (2001). Cultura, política y práctica educativa. Barcelona: Graó.

González, G., \& Barba, J. J. (2013). La perspectiva autobiográfica de un docente novel sobre los aprendizajes de Educación Física en diferentes nieles educativos. CCD, Cultura, Ciencia y Deporte, 8(24), 171-181. Recuperado de http://www.redalyc.org/html/1630/163028753002/

González, G., \& Barba, J. J. (2014). Formación permanente y desarrollo de la identidad reflexiva del profesorado desde las perspectivas grupal e individual. Profesorado: Revista de currículum y formación del profesorado, 18(1), 397-412. Recuperado de http://www.ugr.es/local/recfpro/ rev181COL12.pdf

Grundy, S. (1998). Producto o praxis del currículum. Madrid: Morata.

Habermas, J. (1982). Conocimiento e interés. Madrid: Taurus.

Hamodi, C., López-Pastor, A. T., \& López-Pastor, V. (2014). Red de evaluación formativa y compartida en docencia universitaria: creación, consolidación y líneas de trabajo. Revista de evaluación educativa, 3(1). Recuperado de http://uvadoc.uva.es/handle/10324/10374

Hamodi, C., López-Pastor, A. T., \& López-Pastor, V. (2017). If I experience formative assessment whilst studying at university, will I put it into practice later as a teacher? Formative and shared assessment in Initial Teacher Education (ITE). European Journal of Teacher Education, 40(2), 171-190. doi:10.1080/02619768.2017.1281909

Hernández, J. L., \& Velázquez, R. (Coords.). (2004). La evaluación en educación física. Investigación y práctica en el ámbito escolar. Barcelona: GRAÓ.

Herranz, M. (2013). Desarrollo de procesos de autoevaluación y evaluación compartida en la etapa de Educación Primaria. Un estudio de casos en el área Educación Física (Tesis Doctoral). Universidad de Valladolid, Segovia. Recuperado de http://uvadoc.uva.es/handle/10324/4527

Jiménez, F., \& Navarro, V. (2008). Evaluación formativa y metaevaluación en Educación Física: Dos estudios de casos colectivos en las etapas de educación primaria y secundaria. Revista Española de Educación Física y Deportes, 9. Julio-diciembre, 14-25.

Johnson, D., Johnson, R., \& Holubec, E. (1999). Los nuevos círculos del aprendizaje. La cooperación en el aula y la escuela. Buenos Aires: Aique.

Josso, M. C. (2014). Proceso autobiográfico de (trans) formación identitaria y de conocimiento de sí. Revista mexicana de investigación educativa, 19(62), 735-761. Recuperado de http://www. scielo.org.mx/scielo.php?pid=S1405-66662014000300005\&script=sci_arttext

Latorre, A., Del Rincón, D., \& Arnal, J. (1996). Bases Metodológicas de la Investigación Educativa. Barcelona: Labor.

López-Górriz, I. (2007). Co-construyendo un nuevo paradigma que haga emerger la vida: educación existencial, autobiografía y método. Diálogos. Educación existencial, la autobiografía y el método, 3(52), 29-36.

López-Pastor, V. M. (1999). Prácticas de evaluación en Educación Física: estudio de casos en primaria, secundaria y formación del profesorado. Valladolid: Universidad de Valladolid. Secretariado de publicaciones.

López-Pastor, V. M. (Coord.). (2006). La Evaluación en Educación Física. Revisión de los modelos tradicionales y planteamiento de una alternativa: la evaluación formativa y compartida. Buenos Aires: Miño y Dávila.

López-Pastor, V. M. (Coord.). (2009). La Evaluación Formativa y Compartida en Docencia Universitaria: propuestas, técnicas, instrumentos y experiencias. Madrid: Narcea.

López-Pastor, V. M. (2013). Nuevas perspectivas sobre Evaluación en Educación Física. Revista de Educación Física, 29(3), 4-13. Recuperado de https://www.researchgate.net/profile/Victor_ Pastor/publication/259117123_Nuevas_perspectivas_sobre_evaluacion_en_educacion_fisica/ links/0deec52a0322686759000000.pdf 
López-Pastor, V. M., García, J.M., García Peñuela A., González, M., López, E., Monjas, R., . . . Pérez, D. (2005). ¿Cuándo tenemos poco prestigio? Mirando la viga en el ojo propio. Causas internas y reflexiones del estatus de educación física. RETOS. Nuevas tendencias en Educación Física, Deporte y Recreación, 8, 11-18. Recuperado de http://www.redalyc.org/ $\mathrm{html} / 3457 / 345732273002 /$

López-Pastor, V. M., Kirk, D., Lorente-Catalán, E., MacPhail, A., \& Macdonald, D. (2013). Alternative Assessment in Physical Education: A Review of International Literature. Sport, Education \& Society, 18(1), 57-76. doi:10.1080/13573322.2012.713860

López-Pastor, V. M., Monjas, R., Manrique, J. C., Barba, J. J., \& González-Pascual, M. (2008). Implicaciones de la evaluación en los enfoques de educación Física cooperativa. El papel de la evaluación formativa y compartida en la necesaria búsqueda de la coherencia. Revista Cultura y Educación, 20(4), 457-477. doi:10.1174/113564008786542208

López-Pastor, V. M., Monjas, R., \& Pérez, D. (2003). Buscando alternativas a la forma de entender y practicar la Educación Física Escolar. Barcelona: Inde.

López-Pastor, V. M., Pérez, D., \& García-Peñuela, A. (2001). La capacidad físico-deportiva ¿Reflejo directo de la capacidad profesional?: Algunas reflexiones sobre las ilógicas prácticas evaluativos en la formación y selección del profesorado de Educación Física. Revista Tandem, 5, 77-89.

López-Pastor, V. M., \& Pérez-Pueyo, A. (Coords.). (2017). Evaluación formativa y compartida en educación: experiencias de éxito en todas las etapas educativas. León: Universidad de León. Recuperado de http://buleria.unileon.es/handle/10612/5999

López-Pastor, V. M., Ruano, C., Hernangómez, Á., Cabello, A. M., Hernández, B., Sánchez, B., . . . García, G. (2016). Veinte años de formación permanente del profesorado, investigaciónacción y programación por dominios de acción motriz. RETOS. Nuevas Tendencias en Educación Física, Deporte y Recreación, 29, 270-279. Recuperado de http://www.redalyc.org/ $\mathrm{html} / 3457 / 345743464050 /$

Margalef, L. (2014). Evaluación formativa de los aprendizajes en el contexto universitario: Resistencias y paradojas del profesorado. Educación XXI, 17(2), 35-55. doi:10.5944/educxx1.17.2.11478

Méndez, A. (2005). Hacia una evaluación de los aprendizajes consecuente con los modelos alternativos de iniciación deportiva. Tándem: Didáctica de la Educación Física, 17, 38-58.

McLaren, P. (2005). La vida en las escuelas. Una introducción a la pedagogía crítica en los fundamentos de la educación. Buenos Aires, Argentina: Siglo XXI.

Molina, M., \& López-Pastor, V. M. (2017). La transferencia de la Evaluación Formativa y Compartida desde la Formación Inicial del Profesorado de Educación Física a la práctica real en Educación Primaria. Infancia, Educación y Aprendizaje, 3(2), 626-631. Recuperado de http://revistas.uv.cl/ index.php/IEYA/index

Moreno-Murcia, J. A., Vera, J. A., \& Cervelló, E. (2009). Efectos de la cesión de responsabilidad de la evaluación en la motivación y la competencia percibida en el aula de Educación Física. Revista de Educación, 348, 423-440.

Murillo, F.J., \& Hidalgo, N. (2015). Enfoques Fundamentantes de la Evaluación de Estudiantes para la Justicia Social. Revista Iberoamericana de Evaluación Educativa, 8(1), 43-61. doi:10.15366/ riee

Palacios, A., \& López-Pastor, V. M. (2013). Haz lo que yo digo, pero no lo que yo hago: sistemas de evaluación del alumnado en la formación inicial del profesorado. Revista de Educación, 361, 279-305. Recuperado de https://recyt.fecyt.es/index.php/Redu/article/view/9476

Pascual, C. (2003). La historia de vida de una educadora de profesores de Educación Física: su desarrollo personal y profesional. Ágora para la Educación Física y el Deporte, 2-3, 23-38. Recuperado de http://www5.uva.es/agora/revista/2/agora2_2_carminabanos.pdf

Pérez, L., \& González, D. A. (2011). "Dime cómo evalúas y te diré qué enseñas". Un análisis teórico sobre las relaciones entre la evaluación del aprendizaje y la enseñanza-aprendizaje de la justicia social. Revista Iberoamericana de Evaluación Educativa, 4(1), 135-148. Recuperado de http:// 
hdl.handle.net/10486/661640

Pérez-Pueyo, A. (2005). Estudio del planteamiento actitudinal del área de Educación Física de la Educación Secundaria Obligatoria en la LOGSE: Una propuesta didáctica centrada en una metodología basada en actitudes (Tesis doctoral). Universidad de León, León.

Pérez-Pueyo, A. (2015). La Evaluación Formativa y Compartida en el Marco del Estilo Actitudinal. Revista des-encuentros, 10(1), 6-17. Recuperado de http://cenda.edu.co/revistadesencuentros/ index.php/journal/article/view/54

Pujadas, J. J. (1992). El método biográfico: el uso de las historias de vida en Ciencias Sociales. Madrid: Centro de Investigaciones Sociológicas.

Pujolàs, P. (2008). El aprendizaje cooperativo. 9 ideas clave. Barcelona: Graó.

Rifkin, J. (2010). La civilización empática: la carrera hacia una conciencia global en un mundo en crisis. Madrid: Ediciones Paidós Ibérica.

Ruíz, J. I. (2012). Metodología de la investigación cualitativa (Vol. 15). Bilbao: Universidad de Deusto.

Ruíz-Omeñaca, J.V. (Coord.). (2017). Aprendizaje cooperativo en educación física. De la teoría a la práctica en situaciones motrices. Madrid: CCS.

Sanmartí, N. (2007). 10 ideas clave. Evaluar para aprender. Barcelona: Graó.

Santos-Guerra, M. A. (2003). Una flecha en la diana: La evaluación como aprendizaje. Madrid: Narcea.

Santos-Guerra, M. A. (2015). La evaluación como aprendizaje. Madrid: Narcea.

Santos-Rodríguez, L., \& Fernández-Río, J. (2009). El "Cuaderno de Bitácora" de Educación Física. Elemento central de una propuesta de Metaevaluación. RETOS. Nuevas tendencias en Educación Física, Deporte y Recreación, 16, 92-96. Recuperado de https://recyt.fecyt.es/index.php/retos/ article/view/34982

Sañudo, L.E. (2006). La ética y la investigación educativa. Hallazgos, 6, 83-97. doi:10.15332/s17943841.2006.0006.05

Sebastiani, E. (1993). La evaluación de la Educación Física en la reforma educativa. Apunts Educación Física y Deporte, 31, 17-26.

Sicilia, A., Delgado, M. A., Sáez, P., Manzano, J. I., Varela, R., Cañadas, J. F., \& Gutiérrez, M. (2006). La evaluación de aprendizajes en Educación Física. Diferencias en función del nivel educativo. Motricidad: European Journal of human movement, 17, 71-95. Recuperado de http:// www.redalyc.org/html/2742/274220442006/

Sparkes, A., \& Devís, J. (2007). Investigación narrativa y sus formas de análisis: una visión desde la EF y el deporte. En W. Moreno y S. M. Pulido (Eds.), Educación, cuerpo y ciudad: el cuerpo en las interacciones e instituciones sociales (pp. 43-68). Medellín: Funámbulos.

Torres, J. (2007). Educación en tiempos de neoliberalismo. Madrid: Morata

UNESCO. (2015). Repensar la educación. ¿Hacia un bien común universal? París: UNESCO.

Velázquez-Callado, C. (2006). Evaluando un programa de Educación Física para la paz. En V. M. López-Pastor (Coord.), La evaluación en Educación Física. Revisión de los modelos tradicionales y planteamiento de una alternativa: la evaluación formativa y compartida (pp. 387-397). Buenos Aires: Miño y Dávila.

Velázquez-Callado, C. (2010). Aprendizaje cooperativo en Educación Física. Fundamentos y aplicaciones prácticas. Barcelona: INDE.

Velázquez, R., \& Hernández, J.L. (2005). La educación física y su imagen social a la luz de sus prácticas de evaluación y calificación del aprendizaje. Tándem: Didáctica de la Educación Física, 17, 7-20. 